\title{
Estabilidad polinomial para un sistema acoplado de ecuaciones de onda
}

\section{Andrés Guardia Cayd y Alfonso Pérez Salvatierrd ${ }^{2}$}

Resumen: En este artículo, investigamos el comportamiento asintótico de las soluciones para un sistema acoplado de dos ecuaciones de onda. Una de estas ecuaciones es conservativo y la otra tiene disipación friccional. Demostraremos que el correspondiente semigrupo no es exponencialmente estable. En este caso demostramos que el semigrupo es polinomialmente estable como $t^{-1 / 2}$ en el caso de condiciones de frontera Dirichlet. Adicionalmente, demostramos que la tasa de decaimiento es óptima.

Palabras clave: sistema acoplado; falta de estabilidad exponencial; estabilidad polinomial; tasa de decaimiento óptimo.

\section{Polynomial stability for a coupled system of wave equations}

\begin{abstract}
In this paper, we investigate the asymptotic behavior of the solutions for a coupled system of two wave equations. One of these equations is conservative and the other has frictional damping. We show that the corresponding is not exponentially stable. In this case we prove that the corresponding semigroup is polynomially stable as $t^{-1 / 2}$ in case of Dirichlet boundary condition. Additionally, we show that the rate of decay is optimal.
\end{abstract}

Keywords: coupled system; lack of exponential stability; polynomial stability; optimal decay rate.

Recibido: 17/05/2021. Aceptado: 10/06/2021. Publicado online: 30/06/2021. con revistapesquimat.matematica@unmsm.edu.pe

\footnotetext{
${ }^{1}$ UNMSM, Facultad de Ciencias Matemáticas, e-mail: aguardiac@unmsm.edu.pe
}

${ }^{2}$ UNMSM, Facultad de Ciencias Matemáticas, e-mail: aperezs@unmsm.edu.pe 


\section{Introducción}

En el presente artículo vamos a establecer la estabilidad polinomial y la tasa óptima de decaimiento del $C_{0}$-semigrupo asociado al siguiente sistema acoplado de ecuaciones de onda formulado por el siguiente modelo:

$$
\begin{aligned}
u_{t t}-\Delta u+u_{t}+\alpha(u-v) & =0 \text { en } \Omega \times] 0, \infty[ \\
v_{t t}-\Delta v-\alpha(u-v) & =0 \text { en } \Omega \times] 0, \infty[
\end{aligned}
$$

con condiciones iniciales

$$
\begin{gathered}
u(x, 0)=u_{0}(x), u_{t}(x, 0)=u_{1}(x) \text { en } \Omega \\
v(x, 0)=v_{0}(x), v_{t}(x, 0)=v_{1}(x) \text { en } \Omega .
\end{gathered}
$$

y condiciones de frontera

$$
u=v=0 \text { sobre } \Gamma \times] 0, \infty[
$$

Donde $\Omega$ es un conjunto abierto y acotado de $\mathbb{R}^{n}, n \geq 1$, con frontera $\Gamma=\partial \Omega$ bien regular y $\alpha$ es una constante real positiva.

El modelo dado en (1)-(4), puede usarse para describir la evolución de un sistema que consta de dos membranas elásticas sujetas a una fuerza elástica que atrae una membrana a la otra con un coeficiente $\alpha>0$. El término $u_{t}$, actúa en la primera membrana como estabilizador, para mas detalles al respecto, ver [7].

Los términos disipativos internos, ayudan bastante para que la energía de la ecuación tenga un comportamiento asintótico, como también los efectos térmicos o la memoria actúan como términos disipativos, es de observar, cuando se tiene un sistema de dos ecuaciones de tipo ondas, se requerirá de dos términos disipativos uno en cada ecuación para asegurar un decaimiento exponencial; pero si solo tiene un solo término disipativo interno en una de las ecuaciones, es posible que tengan solo un decaimiento polinomial. En [3, F. Alabau et al., trabajaron un sistema acoplado de dos ecuaciones de ondas con un solo término disipativo en forma general dado por,

$$
\mid \begin{gathered}
\left.u_{t t}-A_{1} u+B u_{t}+\alpha v=0 \text { en } \Omega \times\right] 0, \infty[ \\
\left.v_{t t}-A_{2} v+\alpha v=0 \text { en } \Omega \times\right] 0, \infty[ \\
u=v=0 \text { sobre } \Gamma \times] 0, \infty[ \\
u(x, 0)=u_{0}(x), u_{t}(x, 0)=u_{1}(x), x \in \Omega \\
v(x, 0)=v_{0}(x), v_{t}(x, 0)=v_{1}(x), x \in \Omega
\end{gathered}
$$

Donde $A_{1}, A_{2}$ y $B$ son operadores lineales auto-adjuntos positivos, $B$ operador acotado.

Los autores muestran que el sistema acoplado (6) es disipativo; pero el semigrupo correspondiente no es exponencialmente estable. Además, prueban que la solución del sistema anterior decae polinomialmente a cero a medida que el tiempo tiende al infinito.

M.L. Santos et al. [11], estudian un sistema muy peculiar con respecto al sistema (6), en el que aplicando el método de la energía prueban el decaimiento polinomial de la energía y por ende de la solución.

Motivado por el estudio del sistema (6), planteamos nuestro estudio del sistema enunciado en la introducción.

Naturalmente, no es difícil demostrar que el sistema acoplado en estudio es disipativo. En tal sentido realizando algunos cálculos se puede establecer que la energía de las soluciones del sistema (1)-(2) sujeto a las condiciones iniciales (3)-(4) y condiciones de frontera (5) está dada por

$$
E(t):=\frac{1}{2} \int_{\Omega}\left|u_{t}\right|^{2} d x+\frac{1}{2} \int_{\Omega}\left|v_{t}\right|^{2} d x+\frac{1}{2} \int_{\Omega}|\nabla u|^{2} d x+\frac{1}{2} \int_{\Omega}|\nabla v|^{2} d x+\frac{\alpha}{2} \int_{\Omega}|u-v|^{2} d x
$$


el cual es decreciente para todo $t \geq 0$, desde que

$$
\frac{d}{d t} E(t)=-\int_{\Omega}\left|u_{t}\right|^{2} d x \leq 0 .
$$

En cuanto se refiera a la existencia y unicidad del sistema acoplado en estudio, ver [6]. Nuestro objetivo en el desarrollo de este trabajo es demostrar la falta de estabilidad exponencial, en consecuencia sólo se podrá obtener el decaimiento polinomial. Con el fin de establecer este último, aplicaremos los resultados debido a Borichev y Tomilov [2]. Finalmente estableceremos que la tasa de decaimiento polinomial del sistema es óptima.

\section{Preliminares}

Enunciamos resultados esenciales de la teoría de semigrupos lineales, que serán útiles en el desarrollo de este trabajo. Omitimos las demostraciones, sin embargo el lector interesado puede hallar las demostraciones respectivas en [8] y [10].

Definición 1 Sea $X$ un espacio de Banach, $A$ un operador lineal no acotado, $D(\mathcal{A}) \subset X$ y $U_{0} \in X$ un dato inicial. El Problema de Valor Inicial

$$
\mid \begin{aligned}
\frac{d U}{d t} & =\mathcal{A} U, \quad t>0 \\
U(0) & =U_{0}
\end{aligned}
$$

se denomina Problema Abstracto de Cauchy.

Teorema 1 Sea $\mathcal{H}$ un espacio de Hilbert y el operador lineal $A: D(\mathcal{A}) \subset \mathcal{H} \rightarrow \mathcal{H}$ el generador infinitesimal de un semigrupo de clase $C_{0}$.

i) Si $U_{0} \in D(\mathcal{A})$, entonces el problema (9) tiene una única solución $U(t)=S(t) U_{0}$ satisfaciendo

$$
U \in C^{1}([0, \infty) ; \mathcal{H}) \cap C([0, \infty) ; D(\mathcal{A}))
$$

ii) Si $U_{0} \in \mathcal{H}$, entonces el problema (9) tiene una única solución $U(t)=S(t) U_{0}$ satisfaciendo

$$
U \in C([0, \infty) ; \mathcal{H}) \cap C^{1}((0, \infty) ; \mathcal{H}) \cap C((0, \infty) ; D(\mathcal{A}))
$$

Demostración. Ver [8] y [10].

Teorema 2 Sea $X$ un espacio de Hilbert y $\mathcal{A}: D(\mathcal{A}) \subset X \longrightarrow X$ un operador lineal disipativo con $\operatorname{Im}(I-\mathcal{A})=X$. Si $X$ es reflexivo, entonces $\overline{D(\mathcal{A})}=X$.

Demostración. Ver [8].

Definición 2 Un semigrupo $\{S(t)\}_{t \geq 0}$ es exponencialmente estable si existen constantes $\alpha>0$ y $M \geq 1$ tales que

$$
\|S(t)\|_{\mathcal{L}(X)} \leq M e^{-\alpha t}, \quad \forall t \geq 0
$$

El siguiente teorema, debido a Prüss, caracteriza la estabilidad exponencial de un semigrupo $\{S(t)\}_{t \geq 0}$, tal que $\|S(t)\|_{\mathcal{L}(X)} \leq 1$. 
Teorema 3 (Prüss) Sea $\{S(t)\}_{t>0}$ un semigrupo de clase $C_{0}$ en un espacio de Hilbert $\mathcal{H}$ que satisface $\|S(t)\|_{\mathcal{L}(\mathcal{H})} \leq 1$ y A es el generador infinitesimal de $S$. Entonces $S(t)$ es exponencialmente estable si y sólamente si,

$$
i \mathbb{R}=\{i \lambda: \lambda \in \mathbb{R}\} \subset \rho(\mathcal{A})
$$

$y$

$$
\lim _{|\lambda| \rightarrow \infty} \sup \left\|(i \lambda I-\mathcal{A})^{-1}\right\|_{\mathcal{L}(\mathcal{H})}<\infty .
$$

donde $\rho(\mathcal{A})$ es el conjunto resolvente del operador $A$.

Demostración. Ver Prüss [9].

Definición 3 Un semigrupo $\{S(t)\}_{t \geq 0}$ sobre un espacio de Hilbert $\mathcal{H}$ es polinomialmente estable si existen contantes $C>0$ y $\gamma>\overline{0}$ tales que

$$
\|S(t) u\|_{\mathcal{H}} \leq \frac{C}{t^{\gamma}}\|u\|_{\mathcal{H}}, \forall u \in D(\mathcal{A}) .
$$

El siguiente teorema, debido a A. Borichev y Y. Tomilov, caracteriza la estabilidad polinomial de semigrupos $C_{0}$ acotados sobre los espacios de Hilbert.

Teorema 4 (Borichev y Tomilov) Sea $\{S(t)\}_{t \geq 0}$ un $C_{0}$-semigrupo acotado generado por $A$ y definido sobre el espacio de Hilbert $\mathcal{H}$, tal que $i \mathbb{R} \subset \rho(\mathcal{A})$ y $\alpha$ positivo. Entonces

$$
\left\|(i \lambda I-\mathcal{A})^{-1}\right\|_{\mathcal{L}(\mathcal{H})} \leq C|\lambda|^{\alpha}, \forall \lambda \in \mathbb{R} \Leftrightarrow\left\|S(t) \mathcal{A}^{-1}\right\|_{D(\mathcal{A})} \leq \frac{C}{t^{1 / \alpha}}
$$

Esto es, si el operador resolvente está acotado por un polinomio de grado $\alpha$ real positivo entonces el decaimiento polinomial del semigrupo es de la forma $t^{-1 / \alpha}$.

Demostración. Ver Borichev, A., Tomilov, Y. [2]

Para demostrar la tasa óptima de decaimiento, enunciamos el siguiente resultado.

Teorema 5 Sea $\{S(t)\}_{t \geq 0}$ un semigrupo de clase $C_{0}$, de contracciones sobre un espacio de Hilbert $\mathcal{H}$ con generador infinitesimal $A$ tal que $i \mathbb{R} \subset \rho(\mathcal{A})$. Si

$$
\left\|S(t) U_{0}\right\|_{\mathcal{H}} \leq \frac{1}{t^{\gamma}}\left\|U_{0}\right\|_{D(\mathcal{A})}
$$

entonces, para todo $\varepsilon>0$, existe una constante positiva $C_{\varepsilon}$, tal que

$$
\frac{1}{|\lambda|^{\frac{1}{\gamma}+\varepsilon}}\left\|(i \lambda I-\mathcal{A})^{-1}\right\|_{\mathcal{L}(\mathcal{H})} \leq C_{\varepsilon} .
$$

Demostración. Ver Teorema 5.3 en [4].

\section{Existencia y unicidad}

Reescribimos el problema del valor de frontera inicial (1) )-(4) como un sistema de primer orden para $U=\left(u, u_{t}, v, v_{t}\right)^{T}$, donde $T$ se utiliza para denotar la transpuesta. Entonces $U$ satisface

$$
\mid \begin{aligned}
& \frac{d U}{d t}=\mathcal{A} U, \text { para } t>0 \\
& U(0)=U_{0} .
\end{aligned}
$$


donde $U_{0}=\left(u_{0}, u_{1}, v_{0}, v_{1}\right)$ y $\mathcal{A}: D(\mathcal{A}) \subset \mathcal{H} \longrightarrow \mathcal{H}$ es el operador diferencial definido por

$$
\mathcal{A}=\left(\begin{array}{crcc}
0 & I & 0 & 0 \\
\Delta-\alpha I & -I & \alpha I & 0 \\
0 & 0 & 0 & I \\
\alpha I & 0 & \Delta-\alpha I & 0
\end{array}\right)
$$

siendo $0, I$, y $\Delta$ los operadores nulo, identidad y Laplaciano respectivamente.

Sea

$$
\mathcal{H}=H_{0}^{1}(\Omega) \times L^{2}(\Omega) \times H_{0}^{1}(\Omega) \times L^{2}(\Omega) .
$$

El espacio $\mathcal{H}$, provisto del siguiente producto interno

$$
\langle U, \widetilde{U}\rangle_{\mathcal{H}}=\int_{\Omega} \nabla u \cdot \bar{\nabla} \widetilde{u} d x+\int_{\Omega} \varphi \overline{\widetilde{\varphi}} d x+\int_{\Omega} \nabla v \cdot \bar{\nabla} \widetilde{v} d x+\int_{\Omega} \psi \overline{\widetilde{\psi}} d x+\alpha \int_{\Omega}(u-v) \overline{(\widetilde{u}-\widetilde{v})} d x
$$

Con $U=(u, \varphi, v, \psi), \widetilde{U}=(\widetilde{u}, \widetilde{\varphi}, \widetilde{v}, \widetilde{\psi}) \in \mathcal{H}$; es un espacio de Hilbert, con la norma dada por

$$
\|U\|_{\mathcal{H}}^{2}=\|\nabla u\|_{L^{2}(\Omega)}^{2}+\|\varphi\|_{L^{2}(\Omega)}^{2}+\|\nabla v\|_{L^{2}(\Omega)}^{2}+\|\psi\|_{L^{2}(\Omega)}^{2}+\alpha\|u-v\|_{L^{2}(\Omega)}^{2} .
$$

El dominio del operador $\mathcal{A}$ es el conjunto sobre el cual el operador está bien definido sobre el espacio de fase, esto es,

$$
D(\mathcal{A})=\{U \in \mathcal{H} \mid \mathcal{A} U \in \mathcal{H}\}
$$

Teniendo en cuenta el operador $\mathcal{A}$ definido por (11) y el vector $U=(u, \varphi, v, \psi)$ se deduce

$$
D(\mathcal{A})=\left[\left(H^{2}(\Omega) \cap H_{0}^{1}(\Omega)\right) \times H_{0}^{1}(\Omega)\right]^{2}
$$

No es difícil ver que $\mathcal{A}$ es un operador disipativo en el espacio $\mathcal{H}$ y que $0 \in \rho(\mathcal{A})$. Mas precisamente se tiene

$$
\frac{d}{d t} E(t)=-\int_{\Omega}|\varphi|^{2} d x \leq 0, \forall t \geq 0 .
$$

Por tanto, del teorema de Lummer Phillips tenemos que $\mathcal{A}$ es el generador infinitesimal de un semigrupo de contracciones $\{S(t)\}_{t \geq 0}$ de clase $C_{0}$ sobre el espacio de Hilbert $\mathcal{H}$.

En el siguiente teorema establecemos el resultado sobre existencia y unicidad de soluciones.

Teorema 6 Si $U_{0} \in D(\mathcal{A})$, es decir, $u_{0}, v_{0} \in H^{2}(\Omega) \cap H_{0}^{1}(\Omega)$ y $u_{1}, v_{1} \in H_{0}^{1}(\Omega)$, entonces el problema 10 posee una única solución $U(t)=S(t) U_{0}$ tal que

$$
U \in C\left(\mathbb{R}^{+} ; D(\mathcal{A})\right) \cap C^{1}\left(\mathbb{R}^{+} ; \mathcal{H}\right) .
$$

esto es,

$$
u(t), v(t) \in H^{2}(\Omega) \cap H_{0}^{1}(\Omega), \quad u_{t}(t), v_{t}(t) \in H_{0}^{1}(\Omega) \text { y } u_{t t}(t), v_{t t}(t) \in L^{2}(\Omega) .
$$

Demostración. La demostración, se sigue a partir del Teorema 1

\section{Falta de estabilidad exponencial}

En esta sección vamos a demostrar que el operador resolvente no es uniformemente acotado. Esto significa afirmar que el semigrupo asociado al sistema (1)-(4), no es exponencialmente estable. A fin de demostrar la falta de estabilidad exponencial del sistema mencionado, haremos uso del siguiente resultado. 
Teorema 7 (Gearhart) Sea $S(t)=e^{\mathcal{A} t}$ un $C_{0}$-semigrupo de contracciones sobre un espacio de Hilbert $\mathcal{H}$. Entonces $S(t)$ es exponencialmente estable si y sólo si:

i) $i \mathbb{R} \equiv\{i \lambda: \lambda \in \mathbb{R}\} \subset \rho(\mathcal{A}) y$

ii) $\lim _{|\lambda| \rightarrow \infty} \sup \left\|(i \lambda I-\mathcal{A})^{-1}\right\|_{\mathcal{H}}<\infty$.

Donde $\rho(\mathcal{A})$ es el conjunto resolvente del operador $\mathcal{A}$.

Demostración. Ver [5].

Para abordar el comportamiento asintótico del sistema acoplado de ecuaciones de onda (1)-(4), consideramos el siguiente problema espectral:

$$
\left\{\begin{array}{rccll}
-\Delta w_{m} & = & \lambda_{m} w_{m} & \text { en } & \Omega \\
w_{m} & = & 0 & \text { sobre } & \Gamma
\end{array}\right.
$$

donde $\left(\lambda_{m}\right)_{m \in \mathbb{N}}$ es creciente y

$$
\lim _{m \rightarrow \infty} \lambda_{m}=+\infty
$$

El siguiente Teorema, describe el resultado de esta sección.

Teorema 8 Sea $S(t)$ un $C_{0}$ semigrupo de contracciones generado por $\mathcal{A}$. Entonces $S(t)$ no es exponencialmente estable.

Demostración. En efecto, sea $F=\left(f_{1}, f_{2}, f_{3}, f_{4}\right) \in \mathcal{H}$ y $U=(u, \varphi, v, \psi)$. Consideremos la ecuación resolvente

$$
i \lambda U-\mathcal{A} U=F, \text { con } \lambda \in \mathbb{R} .
$$

De (18) en términos de sus componentes, obtenemos

$$
\begin{aligned}
i \lambda u-\varphi & =f_{1} \\
i \lambda \varphi-\Delta u+\alpha u-\alpha v+\varphi & =f_{2} \\
i \lambda v-\psi & =f_{3} \\
i \lambda \psi-\Delta v-\alpha u+\alpha v & =f_{4}
\end{aligned}
$$

En (19)- -22 considerando las siguientes identidades

$$
f_{1}=f_{3}=0 \quad \text { y } f_{2}=f_{4}=w_{m},
$$

obtenemos el siguiente sistema de ecuaciones

$$
\begin{aligned}
i \lambda u & =\varphi \\
i \lambda \varphi-\Delta u+\alpha u-\alpha v+\varphi & =w_{m} \\
i \lambda v & =\psi \\
i \lambda \psi-\Delta v-\alpha u+\alpha v & =w_{m} .
\end{aligned}
$$

A partir de este último sistema, debemos hallar soluciones de la siguiente forma:

$$
u=a w_{m}, \varphi=b w_{m}, v=c w_{n} \quad \text { y } \quad \psi=d w_{m}
$$


con $a, b, c, d \in \mathbb{C}$, los que serán determinados en forma explícita.

Sustituyendo (23) y (27) en (24) convenientemente y aplicando el problema espectral (17), obtenemos

$$
-\lambda^{2} a w_{m}+\underbrace{\left(-a \Delta w_{m}\right)}_{:=a \lambda_{m} w_{m}}+\alpha a w_{m}-\alpha c w_{n}+b w_{m}=w_{n}
$$

de modo similar, reemplazando (25) y (27) en (26) y aplicando nuevamente (17), obtenemos

$$
-\lambda^{2} c w_{n}+\underbrace{\left(-c \Delta w_{n}\right)}_{:=c \lambda_{m} w_{m}}-\alpha a w_{n}+\alpha c w_{n}=w_{n}
$$

Sumando (28) y (29), se obtiene

$$
-\lambda^{2}(a+c) w_{m}+(a+c) \lambda_{m} w_{m}+b w_{m}=2 w_{n}
$$

Eligiendo $\lambda=\sqrt{\lambda_{m}}$ y sustituyendo en $\sqrt{30}$, se obtiene $b=2$.

Teniendo en cuenta que $u=a w_{m}, \varphi=b w_{m}$ y $b=2$, sustituyendo en (19), obtenemos

$$
i \lambda\left(a w_{m}\right)-2 w_{m}=0
$$

de donde

$$
a=\frac{-2 i}{\sqrt{\lambda_{m}}}
$$

De modo similar en 20 con $v=c w_{n}, u=a w_{m}, \varphi=b w_{m}$, se obtiene

$$
c=\frac{1}{\alpha}-\frac{2 i}{\sqrt{\lambda_{m}}} \quad \mathrm{y} \quad d=\frac{\sqrt{\lambda_{m}} i}{\alpha}+2 .
$$

Por tanto,

$$
u=\frac{-2 i}{\sqrt{\lambda_{m}}} w_{m}, \quad \varphi=2 w_{m}, \quad v=\left(\frac{-2 i}{\sqrt{\lambda_{m}}}+\frac{1}{\alpha}\right) w_{m}, \quad \psi=\left(2+\frac{\sqrt{\lambda_{m}}}{\alpha} i\right) w_{m}
$$

Sólo resta demostrar que $\|U\|_{\mathcal{H}} \rightarrow+\infty$, cuando $m \rightarrow+\infty$.

En efecto, sustituyendo 33 en

$$
\|U\|_{\mathcal{H}}^{2}:=\int_{\Omega}\left(|\nabla u|^{2}+\varphi^{2}+|\nabla v|^{2}+\psi^{2}+\alpha|u-v|^{2}\right) d x
$$

simplificando y teniendo en cuenta que $\lim _{m \rightarrow \infty} \lambda_{m}=+\infty$, se obtiene

$$
\lim _{m \rightarrow \infty}\left\|U_{m}\right\|_{\mathcal{H}}^{2} \geq \lim _{m \rightarrow \infty}\|\psi\|_{L^{2}(\Omega)}^{2}=\lim _{m \rightarrow \infty}\left(4+\frac{\lambda_{m}}{\alpha^{2}}\right)=\infty
$$

Recordando que

$$
i \lambda U-\mathcal{A} U=F \Leftrightarrow U=(i \lambda U-\mathcal{A})^{-1} F,
$$

de (34), 35) y del Teorema 7 se sigue que, el semigrupo $S(t)$ no es exponencialmente estable. Con lo que se concluye la demostración del teorema. 


\section{Decaimiento polinomial}

En esta sección, vamos a demostrar que el semigrupo $S(t)=e^{\mathcal{A} t}$ generado por $\mathcal{A}$, asociado al sistema (1)-(4) tiende a cero polinomialmente conforme transcurre el tiempo, para cualquier condición inicial $U_{0}$ sobre $D(\mathcal{A})$. Para demostrar lo afirmado, haremos uso del resultado debido a A. Borichev y Y. Tomilov. específicamente nos referimos al Teorema 4.

En lo que sigue las tres Proposiciones que presentamos, serán de gran importancia para demostrar nuestro resultado principal.

Proposición 1 Las soluciones del sistema (1)-(4), dada por el Teorema 6, satisface

$$
\int_{\Omega}|\varphi|^{2} d x \leq\|F\|_{\mathcal{H}}\|U\|_{\mathcal{H}}
$$

Demostración. Tomando producto interno en la ecuación resolvente del sistema (1)-(4) sobre $\mathcal{H}$, obtenemos

$$
i \lambda\|U\|_{\mathcal{H}}^{2}-\langle A U, U\rangle_{\mathcal{H}}=\langle F, U\rangle_{\mathcal{H}} .
$$

Desde que $\langle\mathcal{A} U, U\rangle_{\mathcal{H}}=-\int_{\Omega}|\varphi|^{2} d x$, de $[37$ se obtiene

$$
i \lambda\|U\|_{\mathcal{H}}^{2}+\int_{\Omega}|\varphi|^{2} d x=\langle F, U\rangle_{\mathcal{H}}
$$

tomando la parte real y aplicando la desigualdad de Cauchy Schwarz, se concluye

$$
\int_{\Omega}|\varphi|^{2} d x=\operatorname{Re}\langle F, U\rangle \leq\|F\|_{\mathcal{H}}\|U\|_{\mathcal{H}}
$$

Proposición 2 Las soluciones del sistema (1)-(4) dada por el Teorema 6, satisface

$$
\int_{\Omega}|\nabla u|^{2} d x+\int_{\Omega}|\nabla v|^{2}+\alpha \int_{\Omega}|u-v|^{2} d x+\int_{\Omega} \varphi \bar{u} d x \leq M\|U\|_{\mathcal{H}}\|F\|_{\mathcal{H}}+\int_{\Omega}|\psi|^{2} d x
$$

donde $M$ es una constante positiva.

Demostración. En efecto, multiplicando la ecuación 20 y 22 por $\bar{u}$ y $\bar{v}$, respectivamente, e integrando sobre $\Omega$, y luego aplicando el teorema de Green se obtine

$$
\begin{aligned}
& \underbrace{i \lambda \int_{\Omega} \varphi \bar{u} d x}_{:=I_{1}}+\int_{\Omega}|\nabla u|^{2} d x+\int_{\Omega} \varphi \bar{u} d x+\alpha \int_{\Omega}|u|^{2} d x-\alpha \int_{\Omega} v \bar{u} d x \\
& +\underbrace{i \lambda \int_{\Omega} \psi \bar{v} d x}_{:=I_{2}}+\int_{\Omega}|\Delta v|^{2} d x-\alpha \int_{\Omega} u \bar{v} d x+\alpha \int_{\Omega}|v|^{2} d x=\int_{\Omega} f_{2} \bar{u} d x+\int_{\Omega} f_{4} \bar{v} d x .
\end{aligned}
$$

tomando conjugada en (19) y 21), obtenemos

$$
\overline{i \lambda u}=-\left(\bar{\varphi}+\bar{f}_{1}\right), \quad \overline{i \lambda v}=-\left(\bar{\psi}+\bar{f}_{3}\right) .
$$

sustituyendo $\overline{i \lambda u}$ en $I_{1}, \overline{i \lambda v}$ en $I_{2}$, en la relación 41, obtenemos

$$
\begin{aligned}
a \int_{\Omega}|\nabla u|^{2} d x+\int_{\Omega}|\nabla v|^{2} d x+ & \alpha \int_{\Omega}|u-v|^{2} d x \\
& +\int_{\Omega} \varphi \bar{u} d x=\int_{\Omega}|\varphi|^{2} d x+\int_{\Omega}|\psi|^{2} d x+\int_{\Omega} \varphi \overline{f_{1}} d x \\
& +\int_{\Omega} \psi \overline{f_{3}} d x+\int_{\Omega} f_{2} \bar{u} d x+\int_{\Omega} f_{4} \bar{v} d x
\end{aligned}
$$


Luego aplicando la desigualdad de Cauchy Schwarz, Poincaré y teniendo en cuenta que

$$
\int_{\Omega}|\varphi|^{2} d x \leq\|U\|_{\mathcal{H}}\|F\|_{\mathcal{H}}
$$

de (43) se concluye con la demostración.

Proposición 3 La solución fuerte del sistema (1)-(4) dada por el Teorema 6, satisface

$$
\left(1-\frac{C}{|\lambda|}\right) \int_{\Omega}|\psi|^{2} d x \leq \frac{C}{|\lambda|}\left(\int_{\Omega}|\nabla u|^{2} d x+\int_{\Omega}|\nabla v|^{2} d x\right)+K|\lambda|^{2}\|U\|_{\mathcal{H}}\|F\|_{\mathcal{H}}
$$

donde $C$ y $K$ son constantes positivas y $|\lambda|>1$ es lo suficientemente grande.

Demostración. En efecto, multiplicando la ecuación 22 por $\bar{\psi}$ e integrando sobre $\Omega$, obtenemos

$$
i \lambda \int_{\Omega}|\psi|^{2} d x=\int_{\Omega} \Delta v \bar{\psi} d x+\alpha \int_{\Omega} u \bar{\psi} d x-\alpha \int_{\Omega} v \bar{\psi} d x+\int_{\Omega} f_{4} \bar{\psi} d x
$$

tomando conjugada en 21, obtenemos

$$
\bar{\psi}=-\left(i \lambda \bar{v}+\bar{f}_{3}\right)
$$

sustituyendo (46) en (45), aplicando el teorema de Green, Young la desigualdad de Poincaré y la Proposición [1, se obtiene

$$
\left(1-\frac{C}{|\lambda|}\right) \int_{\Omega}|\psi|^{2} d x \leq \frac{C}{|\lambda|}\left(\int_{\Omega}|\nabla u|^{2} d x+\int_{\Omega}|\nabla v|^{2} d x\right)+K|\lambda|^{2}\|U\|_{\mathcal{H}}\|F\|_{\mathcal{H}} .
$$

donde $C$ y $K$ son constantes positivas y $|\lambda|>1$ es lo suficientemente grande.

Ahora, probaremos el resultado principal de este trabajo, el cual enunciamos a continuación.

Teorema 9 El semigrupo $S(t)=e^{\mathcal{A} t}$ asociado al sistema (1)-(4) es polinomialmente estable y

$$
\left\|S(t) U_{0}\right\|_{\mathcal{H}} \leq \frac{\eta}{\sqrt{t}}\left\|U_{0}\right\|_{D(\mathcal{A})} .
$$

Además, este resultado es óptimo.

Demostración. A partir de las proposiciones 2 y 3 y efectuando estimaciones convenientemente se obtiene

$$
\|U\|_{\mathcal{H}} \leq \eta|\lambda|^{2}\|F\|_{\mathcal{H}}, \eta>0 .
$$

Como $(i \lambda I-\mathcal{A}) U=F$ tenemos que

$$
\left\|(i \lambda I-\mathcal{A})^{-1} F\right\|_{\mathcal{H}}=\|U\|_{\mathcal{H}} \leq \eta|\lambda|^{2}\|F\|_{\mathcal{H}}
$$

Entonces

$$
\frac{\left\|(i \lambda I-\mathcal{A})^{-1} F\right\|_{\mathcal{H}}}{\|F\|_{\mathcal{H}}} \leq \eta|\lambda|^{2}
$$

Luego

$$
\left\|(i \lambda I-\mathcal{A})^{-1}\right\|_{\mathcal{L}(\mathcal{H})}=\sup _{\|F\|_{\mathcal{H}} \neq 0}\left(\frac{\left\|(i \lambda I-\mathcal{A})^{-1} F\right\|_{\mathcal{H}}}{\|F\|_{\mathcal{H}}}\right) \leq \eta|\lambda|^{2}
$$


Luego, teniendo en cuenta los resultados debido a Borichev y Tomilov 4 se sigue que

$$
\left\|S(t) \mathcal{A}^{-1}\right\|_{\mathcal{L}(\mathcal{H})}=O\left(t^{-\frac{1}{2}}\right) \quad \text { cuando } t \rightarrow \infty
$$

esto es, existe una constante positiva $\eta$, que satisface

$$
\left\|S(t) \mathcal{A}^{-1} F\right\|_{\mathcal{L}(\mathcal{H})} \leq \frac{\eta}{\sqrt{t}}\|F\|_{\mathcal{H}}
$$

para valores de $t$ suficientemente grande.

Desde que $0 \in \rho(\mathcal{A})$, se sigue que el operador diferencial $\mathcal{A}$ es sobreyectivo sobre $\mathcal{H}$. Por tanto la ecuación $-\mathcal{A} U=F$ posee una única solución $U_{0} \in D(\mathcal{A})$ tal que $\mathcal{A}^{-1} F=U_{0}$. Luego

$$
\begin{aligned}
\left\|S(t) U_{0}\right\|_{\mathcal{H}} & =\left\|S(t) \mathcal{A}^{-1} F\right\|_{\mathcal{H}} \\
& \leq\left\|S(t) \mathcal{A}^{-1}\right\|_{\mathcal{L}(\mathcal{H})}\left\|\mathcal{A} U_{0}\right\|_{\mathcal{H}} \\
& \leq \frac{\eta}{\sqrt{t}}\left\|U_{0}\right\|_{D(\mathcal{A})} .
\end{aligned}
$$

De donde se sigue que

$$
\left\|S(t) U_{0}\right\|_{\mathcal{H}} \leq \frac{\eta}{\sqrt{t}}\left\|U_{0}\right\|_{D(\mathcal{A})} .
$$

para $t$ suficientemente grande, por tanto la solución tiene estabilidad polinomial.

En seguida, demostraremos que la tasa de decaimiento $t^{-1 / 2}$ es la mejor tasa de decaimiento.

En efecto, procediendo por contradicción, supongamos que la tasa $t^{-1 / 2}$ puede ser mejorada por una tasa de la forma $t^{-1 /(2-\varepsilon)}$ con $0<\varepsilon<2$, el cual significa decir que existe una constante $K>0$, tal que

$$
\left\|S(t) \mathcal{A}^{-1}\right\|_{\mathcal{L}(\mathcal{H})} \leq \frac{K}{t^{1 /(2-\varepsilon)}}
$$

entonces por el Teorema 5, para todo $\varepsilon>0$ y $\lambda \in \rho(\mathcal{A})$, el operador

$$
|\lambda|^{-2+\varepsilon}\left\|(i \lambda I-\mathcal{A})^{-1}\right\|_{\mathcal{L}(\mathcal{H})} \leq K, \text { cuando }|\lambda| \rightarrow \infty
$$

el cual implica, que para todo $F \in \mathcal{H}$,

$$
|\lambda|^{-2+\varepsilon}\|U\|_{\mathcal{H}} \leq K\|F\|_{\mathcal{H}}, \forall \lambda \in \mathbb{R}, \lambda \neq 0
$$

donde $U \in D(\mathcal{A})$ es solución de la ecuación resolvente $(i \lambda I-\mathcal{A}) U=F$.

De otro lado teniendo en cuenta el Teorema 8, existe sucesión de números reales $\left\{\lambda_{\mu}\right\}_{\mu \geq 1} \subset \mathbb{R}$ con $\lim _{\mu \rightarrow \infty}\left|\lambda_{\mu}\right|=\infty$ y $\left\{U_{\mu}\right\}_{\mu \geq 1} \subset D(\mathcal{A})$ para $\left\{F_{\mu}\right\}_{\mu \geq 1} \subset \mathcal{H}$, tal que

$$
\left(i \lambda_{\mu} I-\mathcal{A}\right) U_{\mu}=F_{\mu}
$$

es acotado en $\mathcal{H} \mathrm{y}$

$$
\lim _{\mu \rightarrow \infty}|\lambda|^{-2+\varepsilon}\left\|U_{\mu}\right\|_{\mathcal{H}}=\infty
$$

Luego, podemos considerar para cada $\mu \in \mathbb{N}$,

$$
F_{\mu}=\left(0, \omega_{\mu}, 0, \omega_{\mu}\right)^{T}
$$

$\mathrm{y}$

$$
U_{\mu}=\left(u_{\mu}, \varphi_{\mu}, v_{\mu}, \psi_{\mu}\right)^{T}
$$

de donde debido a las condiciones de frontera, $U_{\mu}$ toma la forma

$$
u_{\mu}=a \omega_{\mu}, \varphi_{\mu}=b \omega_{\mu}, v_{\mu}=c \omega_{\mu}, \psi_{\mu}=d \omega_{\mu}
$$


con $a, b, c, d \in \mathbb{C}$.

Luego, siguiendo los mismos argumentos realizados en la demostración del Teorema 8, se concluye

$$
\lim _{\mu \rightarrow \infty}|\lambda|^{-2+\varepsilon}\left\|U_{\mu}\right\|_{\mathcal{H}} \geq O\left(\mu^{\varepsilon}\right) \rightarrow \infty, \text { cuando } \mu \rightarrow \infty .
$$

Por tanto, la tasa de decaimiento polinomial $t^{-1 / 2}$ no puede ser mejorada sobre $D(\mathcal{A})$ y en consecuencia, se concluye con la demostración del Teorema.

\section{Conclusión}

En este artículo consideramos un sistema acoplado de dos ecuaciones de onda débilmente disipativas, que consiste en dos membranas elásticas sometidas a una fuerza elástica, con constante $\alpha$ en ambas ecuaciones. Por otro lado, el estudio del sistema planteado nos indica que con un sólo término disipativo en una de las ecuaciones y con energía asociada al sistema decreciente se obtiene un decaimiento polinomial mas no un decaimiento exponencial.

Así mismo en este trabajo se establece la falta de decaimiento exponencial del sistema en estudio y tomando en cuenta la contribución teórica reciente debido a Borichev y Tomilov, se establece la optimalidad del decaimiento polinómial de la energía asociada al sistema acoplado de dos ecuaciones de onda débilmente disipativo.

\section{Referencias bibliográficas}

[1] Adams, R. A. (1975). Sobolev Spaces. United States of America, New York: Academic Press.

[2] A. Borichev, Y. Tomilov, Optimal polynomial decay of functions and operator semigroups, Math. Ann. Vol. 347(2) (2009) 455-478.

[3] Fatiha Alabau, P. Cannarza and V. Komornik (2002). Indirect internal stabilization of weakly coupled evolution equations. J. Evol. Equ., 2, pp. 127 - 150 .

[4] L. Fatori, J.E.M. Rivera, Rates of decay to weak thermoelastic Bresse system, IMA J. Appl. Math.(2010), 1-24.

[5] L. Gearhart, Spectral theory for contraction semigroups on Hilbert spaces. Trans. AMS 236, 385-394, (1978).

[6] Guardia Cayo, A., \& Pérez Salvatierra, A. (2019). Existencia y unicidad para un sistema acoplado de ecuaciones de onda con término disipativo débil. Pesquimat, 22(2), 75-83. https://doi.org/10.15381/pesquimat.v22i2.172

[7] Love, A. E. H. (1942). Mathematical Theory of Elasticity. Fourth Edition, Dover Publications, New York.

[8] Pazy, A. (1983). Semigroups of Linear Operators and Applications to Partial Differential Equations. Springer-Verlag, New York, NY.

[9] Prüss, J. On the spectrum of $C_{0^{-}}$semigroups. Trans Amer. Math. Soc. 28, 847-857, (1984).

[10] Rivera, J. E. M. (2007). Estabilizacao de Semigrupos e Aplicações. Textos Avanzados. LNCC. Petropolis.

[11] M.L. Santos, S.C. Gomes (2007). On a coupled system of wave equations weakly dissipative, Universidade Federal do Pará, Belén, Brasil. 